\title{
A Note on Nonparametric Estimation of Conditional Hazard Quantile Function
}

\author{
El Hadj Hamel, Nadia Kadiri, Abbes Rabhi \\ Laboratory of Mathematics, University Djillali LIABES of Sidi Bel Abbés, \\ PO. BOX. 89, Sidi Bel Abbés 22000, Algeria \\ E-mail:rbe0222@yahoo.com,nad.kad06@yahoo.com,rabhi_abbes@yahoo.fr
}

Received 14 March 2017

Accepted 18 April 2017

\begin{abstract}
In this paper, we study an kernel estimator of the conditional hazard quantile function (CHQF) of a scalar response variable $Y$ given a random variable (rv) $X$ taking values in a semi-metric space and using the proposed estimator based of the kernel smoothing method. The almost complete consistency and the asymptotic normality of this estimate are obtained when the sample is an independante sequence.
\end{abstract}

Keywords: Asymptotic normality, conditional hazard quantile function, functional data, kernel smoothing, nonparametric estimator.

\section{Introduction}

The goal of this paper is to study a nonparametric estimator of the CHQF when the explanatory variable is functional. This is motivated by the increasing number of situations in which the collected data are curves (consecutive discrete recordings are aggregated and viewed as sampled values of a random curve) where it used to be numbers and vectors. Functional data analysis (see Ferraty and Vieu (2006)) can help to analyze such data sets in a nonparametric framework.

Recently, many authors are interested in the estimation of conditional quantiles for a scalar response and functional covariate. Ferraty et al. (2005) introduced a nonparametric estimator of conditional quantile defined as the inverse of the conditional cumulative distribution function when the sample is considered as an $\alpha$-mixing sequence. They stated its rate of almost complete consistency and used it to forecast the well-known El Ni $\widetilde{n}$ o time series and to build confidence prediction bands. Ezzahrioui et al. (2008) established the asymptotic normality of the kernel conditional quantile estimator under $\alpha$-mixing assumption. Recently, and within the same framework, Dabo-Niang and Laksaci (2012) provided the consistency in $L^{p}$ norm of the conditional quantile estimator for functional dependent data, Bouchentouf et al. (2015) provided the consistency and asymptotic normality of the smoothing conditional quantile density function.

In an earlier contribution of the estimator of the CHQF (see Sankaran and Unnikrishnan (2009)) we established the consistency and asymptotic normality of the kernel smoothing estimator for the independent sequence and reel case. The present work gives a generalization to the functional data, we investigate the asymptotic properties and the asymp- 
totic normality of the CHQF of a scalar response and functional covariate. The interest comes mainly from the fact that application fields for functional methods need to analyze continuous-time stochastic processes.

In what follows, The rest of the paper is organized as follows. Section 2 we present our estimation procedure and recall the definition of the The functional kernel estimates property. Section 3 formulates main results of strong consistency (with rate) and asymptotic normality of the estimator with gives proofs of the main results. Section 4 is devoted provides a brief conclusion of the study.

\section{The functional kernel estimates}

We consider a random pair $(X, Y)$ where $Y$ is valued in $\mathbb{R}$ and $X$ is valued in some infinite dimensional semi-metric vector space $(F, d(\cdot, \cdot))$. Let $\left(X_{i}, Y_{i}\right), i=$ $1, \ldots, n$ be the statistical sample of pairs which are identically distributed like $(X, Y)$,but not necessarily independent. From now on, $X$ is called functional random variable f.r.v. Let $x$ be fixed in $\mathscr{F}$ and let $F_{Y \mid X}(y, x)$ be the conditional cumulative distribution function (cond-cdf) of $Y$ given $X=x$, is defined by:

$$
\forall y \in \mathbb{R}, F_{Y \mid X}(x, y)=\mathbb{P}(Y=y \mid X=x) .
$$

Let $Q_{Y \mid X}(\gamma)$ be the $\gamma$-order quantile of the distribution of $Y$ given $X=x$. From the cond-cdf $F_{Y \mid X}(\cdot, x)$, it is easy to give the general definition of the $\gamma$-order quantile:

$Q(\gamma \mid X=x) \equiv Q_{Y \mid X}(\gamma)=\inf \left\{t: F_{Y \mid X}(t, x)=\gamma\right\}, 0 \leqslant \gamma \leqslant 1$.

Then, the definition of conditional quantile implies that

$$
F_{Y \mid X}\left(Q_{Y \mid X}(\gamma)\right)=\gamma .
$$

On differentiating partially w.r.t. $\gamma$ we get

$$
f_{Y \mid X}\left(Q_{Y \mid X}(\gamma)\right)=\frac{1}{\frac{\partial}{\partial \gamma}\left(Q_{Y \mid X}(\gamma)\right)} .
$$

Thus, the condition quantile density function can be written as follows

$$
q_{Y \mid X}(\gamma)=\frac{1}{f_{Y \mid X}\left(Q_{Y \mid X}(\gamma)\right)} .
$$

Let us now, define the kernel estimator $\widehat{F}_{Y \mid X}(\cdot, x)$ of $F_{Y \mid X}(\cdot, x)$

$$
\widehat{F}_{Y \mid X}(x, y)=\frac{\sum_{i=1}^{n} K\left(h_{K}^{-1} d\left(x, X_{i}\right)\right) H\left(h_{H}^{-1}\left(y-Y_{i}\right)\right)}{\sum_{i=1}^{n} K\left(h_{K}^{-1} d\left(x, X_{i}\right)\right)} .
$$

where $K$ is a kernel function, $H$ a cumulative distribution function and $h_{K}=h_{K, n}\left(\right.$ resp. $\left.h_{H}=h_{H, n}\right)$ a sequence of positive real numbers. Roussas (1969) introduced some related estimate but in the special case when X is real, while Samanta (1980) produced previous asymptotic study. As a by-using of Nair and Sankaran (2009) and Xiang (1995), it is easy to derive an estimator $\widehat{Q}_{Y \mid X}$ of $Q_{Y \mid X}$ :

$$
Q_{Y \mid X}(\gamma)=\inf \left\{t: \widehat{F}_{Y \mid X}(t, x)=\gamma\right\}=F_{Y \mid X}^{-1}\left(Q_{Y \mid X}(\gamma)\right) .
$$

Let now defined the conditional density function is the derivative of conditional distribution function.

$$
\widehat{f}_{Y \mid X}(x, y)=\frac{h_{H}^{-1} \sum_{i=1}^{n} K\left(h_{K}^{-1} d\left(x, X_{i}\right)\right) H\left(h_{H}^{-1}\left(y-Y_{i}\right)\right)}{\sum_{i=1}^{n} K\left(h_{K}^{-1} d\left(x, X_{i}\right)\right)} .
$$

Parzen (1979) and Jones (1992) defined the quantile density function as the derivative of $Q(\gamma)$, that is, $q(\gamma)=Q^{\prime}(\gamma)$. Note that the sum of two quantile density functions is again a quantile density function.

Nair and Sankaran (2009) have defined the hazard quantile function as follows:

$$
r(\gamma)=r(Q(\gamma))=\frac{f(Q(\gamma))}{1-F(Q(\gamma))}=((1-\gamma) q(\gamma))^{-1} .
$$

Thus hazard rate of two populations would be equal if and only if their corresponding quantile density functions are equal. This has been used to construct tests for testing equality of failure rates of two independent samples. Now, from this definition, let 
us introduce the $\gamma$-order conditional quantile of the conditional hazard function

$$
\begin{aligned}
& r(\gamma)=r_{Y \mid X}\left(Q_{Y \mid X}(\gamma)\right)=\frac{f_{Y \mid X}\left(Q_{Y \mid X}(\gamma)\right)}{1-F_{Y \mid X}\left(Q_{Y \mid X}(\gamma)\right)} \\
& =\left((1-\gamma) q_{Y \mid X}(\gamma)\right)^{-1} .
\end{aligned}
$$

Consequently, the conditional quantiles of conditional hazard function operator is defined in a natural way and can be estimated by using kernel smoothing methods by

$$
r_{n}(\gamma)=\widehat{r}_{Y / X}\left(\widehat{Q}_{Y / X}(\gamma)\right)=\frac{\widehat{f}_{Y / X}\left(\widehat{Q}_{Y / X}(\gamma)\right)}{1-\widehat{F}_{Y / X}\left(\widehat{Q}_{Y / X}(\gamma)\right)}
$$

Now we proposed the other estimator of $r_{n}(\gamma)$ using the kernel smoothing method, define by:

$r_{n}(\gamma)=\frac{1}{h_{H}} \int_{0}^{1} \frac{1}{\left[1-\widehat{F}_{Y \mid X}\left(\widehat{Q}_{Y \mid X}(t)\right)\right] \cdot \widehat{q}_{Y \mid X}(t)} H\left(\frac{t-\gamma}{h_{H}}\right) d t$.

In the next section derive the asymptotic properties of our conditional quantile hazard function

\section{Assumptions and main results}

\subsection{General Assumptions}

Our results are stated under some assumptions we gather hereafter for easy reference.

(H1) For all $h>0, \mathbb{P}(X \in B(x, h))=: \phi_{x}(h)>0$. Moreover, $\phi_{x}(h)>0 \longrightarrow 0$ as $h \longrightarrow 0$.

(H2) For all $i \neq j, 0<\sup _{i \neq j} \mathbb{P}\left[\left(X_{i}, X_{j}\right) \in B(x, h) \times\right.$ $B(x, h)]=\mathbb{P}\left(W_{i} \leqslant h, W_{j} \leqslant h\right) \leqslant \psi_{x}(h)$, where $\psi_{x}(h) \longrightarrow 0$ as $h \longrightarrow 0$.

Furthermore, we assume that $\psi_{x}(h)=$ $\mathscr{O}\left(\phi_{2}^{x}(h)\right)$.

(H3) $H$ is such that, for all $\left(y_{1}, y_{2}\right) \in \mathbb{R}^{2}, \mid H\left(y_{1}\right)-$ $H\left(y_{2}\right)|=C| y_{1}-y_{2} \mid$

and its first derivative $H^{(1)}$ verifies $\int|t|^{b_{2}} H^{(1)}(t) d t<\infty$.
(H4) $K$ is a nonnegative bounded kernel of class $C_{1}$ over its support $[0,1]$ such that $K(1)>0$.

The derivative $K^{\prime}$ exists on $[0,1]$ and satisfy the condition $K^{\prime}(t)<0$, for all $t \in[0,1]$ and $\int_{0}^{1}(K)^{j}(t) d t<\infty$ for $j=1,2$.

(H5) $\lim _{n \longrightarrow \infty} h_{K}=0$ with $\lim _{n \longrightarrow \infty} \frac{\log n}{n \phi_{x}\left(h_{K}\right)}=0$.

Remark 1. Hypothesis $(H 1)$ is the classical concentration assumption.(H3) allows to get the convergence rate in the independent case.

Assumption $\mathrm{H} 3$ is classical in nonparametric estimation and is satisfied by usual kernels such as Epanechnikov, Biweight, whereas the Gaussian density $K$ is also possible, it suffices to replace the compact support assumption by: $\int_{\mathbb{R}^{d}}|t|^{b_{2}} H(t) d t<\infty$.

Assumption $H 3$ ensures the existence and uniqueness of the quantile estimate $q_{\gamma}(x)$, see Ferraty et al. (2005).

A mild regularity hypothesis $(H 4)$ is assumed for the distribution function. Hypothesis $(H 3)$ is technical and is imposed only for the brevity of proofs. Finally The choice of bandwidth is given by (H5).

\subsection{Asymptotic properties}

In this section, we prove strong consistency and asymptotic normality of the estimator 7 .

Theorem 1. Let $F_{Y \mid X}$ be continuous.Assume that $K(\cdot)$ satisfies the conditions (H1)-(H5) in Secestimator $r_{n}(\gamma)$ is uniformly strong consistent.

Proof.

We can write Equation (7) as 


$$
\begin{aligned}
r_{n}(\gamma) & =\frac{1}{h_{H}} \int_{0}^{1} H\left(\frac{t-\gamma}{h_{H}}\right) \frac{d t}{\left[1-\widehat{F}_{Y \mid X}\left(\widehat{Q}_{Y \mid X}(t)\right)\right] \cdot \widehat{q}_{Y \mid X}(t)} \\
& -\frac{1}{h_{H}} \int_{0}^{1} H\left(\frac{t-\gamma}{h_{H}}\right) \frac{d t}{\left[1-F_{Y \mid X}\left(Q_{Y \mid X}(t)\right)\right] \cdot \widehat{q}_{Y \mid X}(t)} \\
& +\frac{1}{h_{H}} \int_{0}^{1} H\left(\frac{t-\gamma}{h_{H}}\right) \frac{d t}{\left[1-F_{Y \mid X}\left(Q_{Y \mid X}(t)\right)\right] \cdot \widehat{q}_{Y \mid X}(t)} \\
= & \frac{1}{h_{H}} \int_{0}^{1} H\left(\frac{t-\gamma}{h_{H}}\right) \frac{1}{\widehat{q}_{Y \mid X}(t)}\left[\frac{1}{\left[1-\widehat{F}_{Y \mid X}\left(\widehat{Q}_{Y \mid X}(t)\right)\right]}\right. \\
& +\frac{1}{h_{H}} \int_{0}^{1} H\left(\frac{t-\gamma}{h_{H}}\right) \frac{d t}{\left[1-F_{Y \mid X}\left(Q_{Y \mid X}(t)\right)\right] \cdot \widehat{q}_{Y \mid X}(t)} \\
= & \frac{1}{h_{H}} \int_{0}^{1} H\left(\frac{t-\gamma}{h_{H}}\right) \\
& \frac{\widehat{q}_{Y \mid X}(t)\left[1-F_{Y \mid X}\left(Q_{Y \mid X}(t)\right)\right]}{\left.\left.\left.\widehat{F}_{Y \mid X}(t)\right)\right]\left[1-\widehat{Q}_{Y \mid X}(t) \widehat{Q}_{Y \mid X}(t)\right)\right]} \\
& +\frac{1}{h_{H}} \int_{0}^{1} H\left(\frac{t-\gamma}{h_{H}}\right) \frac{d t}{\left[1-F_{Y \mid X}\left(Q_{Y \mid X}(t)\right)\right] \widehat{q}_{Y \mid X}(t)}
\end{aligned}
$$

Since

$$
\sup _{t}\left|\widehat{F}_{Y \mid X}(t)-F_{Y \mid X}(t)\right| \longrightarrow 0
$$

almost surely, equation (8) is asymptotically equal to

$$
r_{n}(\gamma)=\frac{1}{h_{H}} \int_{0}^{1} H\left(\frac{t-\gamma}{h_{H}}\right) \frac{d t}{\left(1-F_{Y \mid X}\left(Q_{Y \mid X}(t)\right) \widehat{q}_{Y \mid X}(t)\right.} .
$$

Thus,

$$
\begin{aligned}
& r_{n}(\gamma)-r(\gamma) \\
&=\frac{1}{h_{H}} \int_{0}^{1} H\left(\frac{t-\gamma}{h_{H}}\right) \frac{1}{\left(1-F_{Y \mid X}\left(Q_{Y \mid X}(t)\right)\right)} \\
& {\left[\frac{1}{\widehat{q}_{Y \mid X}(t)}-\frac{1}{q_{Y \mid X}(t)}\right] d t+} \\
& \frac{1}{h_{H}} \int_{0}^{1} H\left(\frac{t-\gamma}{h_{H}}\right) \frac{1}{q_{Y \mid X}(t)} \\
& \frac{d t}{\left(1-F_{Y \mid X}\left(Q_{Y \mid X}(t)\right)\right)}-\frac{1}{(1-\gamma) q_{Y \mid X}(\gamma)} \\
&= \frac{1}{h_{H}} \int_{0}^{1} \frac{1}{(1-t)} H\left(\frac{t-\gamma}{h_{H}}\right) \\
& \frac{\left[q_{Y \mid X}(t)-\widehat{q}_{Y \mid X}(t)\right] d t}{\widehat{q}_{Y \mid X}(t) q_{Y \mid X}(t)} d t+\frac{1}{h_{H}} \int_{0}^{1} H\left(\frac{t-\gamma}{h_{H}}\right) \\
& \frac{d t}{\left(1-F_{Y \mid X}\left(Q_{Y \mid X}(t)\right)\right) q_{Y \mid X}(t)}-\frac{1}{(1-\gamma) q_{Y \mid X}(\gamma)}
\end{aligned}
$$

\section{Denoting}

$K^{*}(t, \gamma)=\left(H\left((t-\gamma) / h_{H}\right)\right) /(1-t) \widehat{q}_{Y \mid X}(t) q_{Y \mid X}(t)$,

on using integration by parts, equation (9) reduces to

$$
\begin{aligned}
& r_{n}(\gamma)-r(\gamma) \\
& =\frac{1}{h_{H}} \int_{0}^{1}\left(Q_{Y \mid X}(t)-\widehat{Q}_{Y \mid X}(t)\right) d K^{*}(t, \gamma)+ \\
& \quad \frac{1}{h_{H}} \int_{0}^{1} H\left(\frac{t-\gamma}{h_{H}}\right) \frac{d t}{(1-t) q_{Y \mid X}(t)} \\
& \quad-\frac{1}{(1-\gamma) q_{Y \mid X}(\gamma)} .
\end{aligned}
$$

Since $\sup _{t}\left|\widehat{Q}_{Y \mid X}(t)-Q_{Y \mid X}(t)\right| \longrightarrow 0$ almost surely, equation (10) is asymptotically equal to 


$$
\begin{aligned}
& r_{n}(\gamma)-r(\gamma) \\
& =\frac{1}{h_{H}} \int_{0}^{1} H\left(\frac{t-\gamma}{h_{H}}\right) \frac{d t}{(1-t) q_{Y \mid X}(t)} \\
& \quad-\frac{1}{(1-\gamma) q_{Y \mid X}(\gamma)} .
\end{aligned}
$$

Setting $(t-\gamma) / h_{H}=v$, in equation (10),

$$
\begin{aligned}
r_{n}(\gamma)-r(\gamma) & \\
= & \frac{1}{h_{H}} \int_{-\gamma / h_{H}}^{(1-\gamma) / h_{H}} H(v) \frac{f_{Y \mid X} Q_{Y \mid X}\left(\gamma+v h_{H}\right)}{1-\left(\gamma+v h_{H}\right)} d v \\
& -\frac{1}{(1-\gamma) q_{Y \mid X}(\gamma)} \\
= & \frac{1}{h_{H}} \int_{-\gamma / h_{H}}^{(1-\gamma) / h_{H}} H(v) \frac{1}{1-\gamma}\left[1-\frac{v h_{H}}{1-\gamma}\right]^{-1} \\
& f_{Y \mid X} Q_{Y \mid X}\left(\gamma+v h_{H}\right) d v-\frac{1}{(1-\gamma) q_{Y \mid X}(\gamma)} .
\end{aligned}
$$

By Taylor's series expansion of $Q_{Y \mid X}\left(\gamma+v h_{H}\right)$ around $\gamma$, equation (11) becomes

$$
\begin{aligned}
& r_{n}(\gamma)-r(\gamma) \\
& =\frac{1}{h_{H}(1-\gamma)} \int_{-u / h_{H}}^{(1-\gamma) / h_{H}}\left(H(v)\left[1+\frac{v h_{H}}{1-\gamma}+\cdots \cdots\right]\right. \\
& \left.\quad \times f_{Y \mid X}\left[Q_{Y \mid X}(\gamma)+v h_{H} d Q_{Y \mid X}(\gamma)+\cdots \cdots\right]\right) d v \\
& \quad-\frac{1}{(1-\gamma) q_{Y \mid X}(\gamma)}
\end{aligned}
$$

As $n \longrightarrow \infty$,we have $h_{n} \longrightarrow 0$ and $\int_{-\infty}^{+\infty} H(v) d v=$ 1 , so that equation (12) reduces to

$$
\left|r_{n}(\gamma)-r(\gamma)\right|=\left|\frac{f_{Y \mid X}\left(Q_{Y \mid X}\right)(\gamma)}{1-\gamma}-\frac{1}{(1-\gamma) q_{Y \mid X}(\gamma)}\right|,
$$

which tends to zero as $n \longrightarrow \infty$. This completes the proof.

\subsection{Asymptotic normality}

In this section we give the asymptotic normality of $r_{n}(\gamma)$.

Theorem 2. Under assumptions $(\mathrm{H1})(\mathrm{H} 5)$ and suppose that $F_{Y \mid X}$ is continuous, for $0<\gamma<1$, $\sqrt{n}\left(r_{n}(\gamma)-r(\gamma)\right)$ is asymptotically normal with mean zero and variance $\sigma^{2}(\gamma)$ as given in Equation (13).

$$
\begin{aligned}
& \sigma^{2}(\gamma) \\
& =n \frac{1}{h_{H}^{2}} \mathbb{E}\left[\int_{0}^{1} Q_{Y \mid X}(t) d M^{\prime}(t, \gamma)\right. \\
& \left.\quad+\int_{0}^{1} \widehat{F}_{Y \mid X}\left(\widehat{Q}_{Y \mid X}\right)(t) \frac{M(t, \gamma)}{(1-t)} d Q_{Y \mid X}(t)\right]^{2} .
\end{aligned}
$$

$$
M(t, \gamma)=H\left((t-\gamma) / h_{H}\right) / q_{Y \mid X}(t)
$$

and $M^{\prime}(t, \gamma)$ is the derivative of $M(t, \gamma)$ with respect to $t$.

\section{Proof.}

$$
\begin{aligned}
& \sqrt{n}\left(r_{n}(\gamma)-r(\gamma)\right) \\
= & \sqrt{n} \frac{1}{h_{H}} \int_{0}^{1} H\left(\frac{t-\gamma}{h_{H}}\right) \\
& {\left[\frac{1}{1-\widehat{F}_{Y \mid X}\left(\widehat{Q}_{Y \mid X}\right)(t)} \cdot \frac{1}{\widehat{q}_{Y \mid X}(t)}\right.} \\
& \left.-\frac{1}{\left(1-\widehat{F}_{Y \mid X}\left(\widehat{Q}_{Y \mid X}\right)(t)\right) q_{Y \mid X}(t)}\right] d t \\
& +\sqrt{n} \frac{1}{h_{H}} \int_{0}^{1} H\left(\frac{t-\gamma}{h_{H}}\right) \\
& \frac{d t}{\left(1-\widehat{F}_{Y \mid X}\left(\widehat{Q}_{Y \mid X}\right)(t)\right) q_{Y \mid X}(t)}-\frac{\sqrt{n}}{(1-\gamma) q_{Y \mid X}(t)},
\end{aligned}
$$


which can be written as

$$
\begin{aligned}
& \sqrt{n}\left(r_{n}(\gamma)-r(\gamma)\right) \\
= & \frac{\sqrt{n}}{h_{H}} \int_{0}^{1} H\left(\frac{t-\gamma}{h_{H}}\right) \frac{1}{\left(1-\widehat{F}_{Y \mid X}\left(\widehat{Q}_{Y \mid X}\right)(t)\right)} \\
& {\left[\frac{q_{Y \mid X}(t)-\widehat{q}_{Y \mid X}(t)}{q_{Y \mid X}(t) \widehat{q}_{Y \mid X}(t)}\right] d t } \\
& +\frac{\sqrt{n}}{h_{H}} \int_{0}^{1} H\left(\frac{t-\gamma}{h_{H}}\right) \frac{1}{q_{Y \mid X}(t)} \\
& {\left[\frac{\left(\widehat{F}_{Y \mid X}\left(\widehat{Q}_{Y \mid X}\right)(t)-F_{Y \mid X}\left(Q_{Y \mid X}\right)(t)\right) d t}{\left(1-\widehat{F}_{Y \mid X}\left(\widehat{Q}_{Y \mid X}\right)(t)\right)\left(1-F_{Y \mid X}\left(Q_{Y \mid X}\right)(t)\right)}\right] } \\
& +\frac{\sqrt{n}}{h_{H}} \int_{0}^{1} H\left(\frac{t-\gamma}{h_{H}}\right) \\
& \frac{d t}{\left(1-F_{Y \mid X}\left(Q_{Y \mid X}\right)(t)\right) q_{Y \mid X}(t)}-\frac{\sqrt{n}}{(1-\gamma) q_{Y \mid X}(\gamma)} .
\end{aligned}
$$

Since $\sup _{t}\left|\widehat{F}_{Y \mid X}\left(\widehat{Q}_{Y \mid X}\right)(t)-F_{Y \mid X}\left(Q_{Y \mid X}\right)(t)\right| \longrightarrow 0$ and $F_{Y \mid X}\left(Q_{Y \mid X}\right)(t)=t$, equation (14) is asymptotically equal to

$$
\begin{aligned}
= & \sqrt{n} \frac{1}{h_{H}} \int_{0}^{1} H\left(\frac{t-\gamma}{h_{H}}\right) \frac{\left(q_{Y-X}(t)-\widehat{q}_{Y-X}(t)\right) d t}{(1-t)\left(q_{Y \mid X}(t)\right)^{2}} \\
+ & \frac{\sqrt{n}}{h_{H}} \int_{0}^{1} H\left(\frac{t-\gamma}{h_{H}}\right) \frac{1}{q_{Y \mid X}(t)} \frac{1}{(1-t)^{2}} \\
& {\left[\widehat{F}_{Y \mid X}\left(\widehat{Q}_{Y \mid X}\right)(t)-F_{Y \mid X}\left(Q_{Y \mid X}\right)(t)\right] d t } \\
+ & \sqrt{n} \frac{1}{h_{H}} \int_{0}^{1} H\left(\frac{t-\gamma}{h_{H}}\right) \\
& -\frac{d t}{\left.(1-\gamma) F_{Y \mid X}\left(Q_{Y \mid X}\right)(t)\right) q_{Y \mid X}(t)} .
\end{aligned}
$$

Setting $M(t, \gamma)=H\left((t-\gamma) / h_{H}\right) / q_{Y \mid X}(t)$ in equation (15) and applying integration by parts, we obtain

$$
\begin{aligned}
& \sqrt{n}\left(r_{n}(\gamma)-r(\gamma)\right) \\
&=\frac{\sqrt{n}}{h_{H}} \int_{0}^{1} \frac{M(t, \gamma) q_{Y \mid X}(t)}{(1-t)} \\
& \quad\left[\widehat{F}_{Y \mid X}\left(\widehat{Q}_{Y \mid X}\right)(t)-F_{Y \mid X}\left(Q_{Y \mid X}\right)(t)\right] d t \\
&+\frac{\sqrt{n}}{h_{H}} \int_{0}^{1} H\left(\frac{t-\gamma}{h_{H}}\right) \\
& \frac{d t}{\left(1-F_{Y \mid X}\left(Q_{Y \mid X}\right)(t)\right) q_{Y \mid X}(t)} \\
&- \frac{\sqrt{n}}{(1-\gamma) q_{Y \mid X}(\gamma)} \\
&+ \frac{\sqrt{n}}{h_{H}} \int_{0}^{1}\left(\widehat{Q}_{Y \mid X}(t)-Q_{Y \mid X}(t)\right) d M^{\prime}(t, \gamma),
\end{aligned}
$$

where $M^{\prime}(t, \gamma)$ is the derivative of $M(t, \gamma)$ with respect to $t$. From equation (12), we can obtain equation (16) as

$$
\begin{gathered}
\sqrt{n}\left(r_{n}(\gamma)-r(\gamma)\right)=\frac{\sqrt{n}}{h_{H}} \int_{0}^{1} \frac{M(t, \gamma) q_{Y \mid X}(t)}{(1-t)} \\
\quad\left[\widehat{F}_{Y \mid X}\left(\widehat{Q}_{Y \mid X}\right)(t)-F_{Y \mid X}\left(Q_{Y \mid X}\right)(t)\right] \\
+\frac{\sqrt{n}}{h_{H}} \int_{0}^{1}\left(\widehat{Q}_{Y \mid X}(t)-Q_{Y \mid X}(t)\right) d M^{\prime}(t, \gamma) .
\end{gathered}
$$

Note that from Ezzahrioui and Ould-Saïd (2008), Laksaci et al. (2011) and Chaouch and Khardani (2015) for $0 \leqslant \gamma \leqslant 1, \sqrt{n}\left(\widehat{Q}_{Y \mid X}(\gamma)-Q_{Y \mid X}(\gamma)\right)$ is asymptotically normal with mean zero and variance

$$
\sigma^{2}(\gamma)=\frac{1}{\sqrt{\phi_{x}(h)}} \frac{\beta_{2}}{\beta_{1}^{2}} \frac{\gamma(1-\gamma)}{\left(f_{Y \mid X}\left(Q_{Y \mid X}(\gamma)\right)\right)^{2}},
$$

$\beta_{j}=K^{j}(1)-\int_{0}^{1}\left(K^{j}\right)^{\prime}(s) \tau_{0}(s) d s$,

and $\tau_{0}$ is a nondecreasing bounded function such that, uniformly in $s \in[0,1]$,

$$
\frac{\phi(h s)}{\phi(h)}=\tau_{0}(s)+o(1) \text { as } h \downarrow 0
$$

and for $j \geqslant 1$,

$$
\int_{0}^{1}\left((K)^{j}(t)\right)^{\prime} \tau_{0}(t) d t<\infty .
$$

Thus $\sqrt{n}\left(\widehat{F}_{Y \mid X}\left(\widehat{Q}_{Y \mid X}\right)(\gamma)-F_{Y \mid X}\left(Q_{Y \mid X}\right)(\gamma)\right)$ is also asymptotically normal with mean zero and variance $\sigma_{1}^{2}(\gamma)$, since $d / d \gamma F_{Y \mid X}\left(Q_{Y \mid X}\right)(\gamma)=1$. 
Now from equation (17), we can show that $\sqrt{n}\left(r_{n}(\gamma)-r(\gamma)\right)$ is asymptotically normal with mean zero. The expression of variance can be obtained from equation (17), which is given by

$$
\begin{aligned}
\sigma_{1}^{2}(\gamma) & =\frac{n}{h_{H}^{2}} \mathbb{E}\left[\int_{0}^{1} \widehat{Q}_{Y \mid X}(t) d M^{\prime}(t, \gamma)\right. \\
+ & \left.\int_{0}^{1} \widehat{F}_{Y \mid X}\left(\widehat{Q}_{Y \mid X}\right)(t) \frac{M(t, \gamma)}{(1-t)} d Q_{Y \mid X}(t)\right] .(18)
\end{aligned}
$$

This completes the proof.

\section{Remark 2.}

- The function $\tau_{0}(\cdot)$ defined in by there exists a function $\tau_{0}(\cdot)$ s.t. for all $s \in[0,1]$, $\lim _{r \longrightarrow 0} \phi_{x}(s r) / \phi_{x}(r)=\phi_{x}(s)$, permits to get the variance term explicitly.

This condition is classical and related to a non vanishing conditional density. The second one means that a small amount a concentration is needed in order to ensure asymptotic normality.

- The present study provided a nonparametric estimator, for the conditional hazard quantile function, its based and using the kernel smoothing method, and the asymptotic properties of the kernel estimator were studied. The kernel based estimator seems to perform satisfactorily except for large values of $\gamma$.

\section{References}

1. D. Bosq, Linear Processes in Function Spaces, (Lecture Notes in Statistics 149, Springer-Verlag, Berlin, 2000).

2. A.A. Bouchentouf, Y. Hammou, K. Nedjadi and A. Rabhi, Nonparametric Estimation of a Conditional Quantile Density Function for Time Series Data, $J$. Stat. Appl. Prob. 4(1) (2015), pp. 13-21.

3. M. Chaouch and S. Khardani, Randomly censored quantile regression estimation usinf functional stationary ergodic data, Journal of Nonparametric Statistics, 27 (1) (2015), pp. 65-87.

4. M. Ezzahrioui, and E. Ould-Saïd, Asymptotic results of a nonparametric conditional quantile estimator for functional time series, Comm. Statist. Theory Methods. 37(16-17) (2008), pp. 2735-2759.

5. S. Dabo-Niang, and A. Laksaci, Nonparametric quantile regression estimation for functional dependent data, Comm. Statist. Theory Methods, 41(7) (2012), pp. 1254-1268.
6. F. Ferraty, A. Laksaci, A. Tadj, and P. Vieu, Rate of uniform consistency for nonparametric estimates with functional variables, J. Stat. Plann. and Inf., 140 (2010), pp. 335-352.

7. F. Ferraty, A. Rabhi, and P. Vieu, Conditional Quantiles for Functionally Dependent Data with Application to the Climatic El Ni o Phenomeno, Sankhya: The Indian Journal of Statistics, Special Issue on Quantile Regression and Related Methods, 67(2) (2005), pp. 378-398.

8. F. Ferraty, A. Rabhi, and P. Vieu, Estimation nonparamétrique de la fonction de hasard avec variables explicatives fonctionnelles, Rev: Rom. J. Pure \& Applied Math, 52 (2008), pp. 1-18.

9. F. Ferraty, and P. Vieu, Nonparametric Functional Data Analysis: Theory and Practice, (Springer Series in Statistics, Springer, New York, 2006).

10. M.C. Jones, Estimating densities, quantiles, quantile densities and density quantiles, Ann. Inst. Statist. Math., 44(4) (1992), pp. 721-727.

11. A. Laksaci, M. Lemdani, and E. Ould-Saïd, Asymptotic Results for an $L^{1}$-norm Kernel Estimator of the Conditional Quantile for Functional Dependent Data with Application to Climatology, Sankhya: The Indian Journal of Statistics, 73 (A, Part 1) (2011), pp. 125141.

12. N.U. Nair, and P.G. Sankaran, Quantile based reliability analysis, Communications in Statistics Theory and Methods, 38 (2009), pp. 222-232.

13. G. Roussas, Nonparametric estimation of the transition distribution function of a Markov process, Ann. Math. Statist. 40 (1969), pp. 1386-1400.

14. M. Samanta, Nonparametric estimation of conditional quantiles, J. of Statist. Proba. Lett. 7 (1989), pp. 407 412.

15. P.G. Sankaran, and N. Unnikrishnan, Nonparametric estimation of hazard quantile function, Journal of Nonparametric Statistics, 21(6) (2009), pp. 757-767.

16. R.J. Serfling, Approximation Theorem of Mathematical Statistics, (John Wiley, New York 1980).

17. E. Parzen, Nonparametric statistical data modelling, Journal of American Statistical Association 74 (1979), pp. 105-122.

18. J.O. Ramsay, and C.J. Dalzell, Some tools for functional data analysis (with discussion), J. Roy. Statist. Soc., Ser. B (1991), pp. 539-572.

19. J.O. Ramsay, and B.W. Silverman, Applied functional data analysis: Methods and Case Studies, (Springer Series in Statistics, Springer-Verlag, New York, 2002).

20. J.O. Ramsay, and B.W. Silverman, Functional data analysis, ( $2^{\text {nd }}$ Ed.), (Springer Series in Statistics, Springer-Verlag, New-York, 2006).

21. X. Xiang, Estimation of conditional quantile density function, Journal of Nonparametric Statistics, 4 (1995), pp. 309-316. 